\title{
Gold Nanoparticles: Acceptors for Efficient Energy Transfer from the Photoexcited Fluorophores
}

\author{
Debanjana Ghosh, Nitin Chattopadhyay* \\ Department of Chemistry, Jadavpur University, Kolkata, India \\ Email: "nitin.chattopadhyay@yahoo.com
}

Received November 14, 2012; revised December 15, 2012; accepted December 22, 2012

\begin{abstract}
The citrate reduction method of synthesis of gold nanoparticles (AuNP) is standardized with the assistance of instruments like spectrophotometer and TEM. A correlation has been developed between the particle diameter and the fractional concentration of the reductant. This enables one to assess the diameter of the AuNP to be synthesized, in advance, from the composition of the reaction mixture and the diameter of the synthesized particles can be confirmed simply from spectrophotometry. Further, it has been demonstrated that the synthesized AuNPs serve as excellent acceptors for a super-efficient energy transfer (ET) from the donor coumarin 153, leading to a quenching of fluorescence of the latter. The Stern-Volmer constants determined from the fluorescence lifetimes are in the range $10^{7}-10^{9} \mathrm{~mol}^{-1} \cdot \mathrm{dm}^{3}$ and are orders of magnitude higher than the normal photochemical quenching processes. The energy transfer efficiency increases radically with an increase in the size of the metal nanoparticle. The highly efficient energy transfer and the variation of the efficiency of the ET process with a variation of the particle size is ascribed to a large enhancement in the extinction coefficient and an increase in the spectral overlap between the plasmon absorption band of AuNPs and the fluorescence spectrum of $\mathrm{C} 153$ with an increase in the size of the nanoparticles. The impact of the work remains in providing a demonstration of a super quenching effect of the AuNPs and projects that they can be exploited for developing biosensors with high degree of sensitivity, if tagged to the biomacromolecules.
\end{abstract}

Keywords: Gold Nanoparticles; TEM; Spectrophotometry; Fluorescence Quenching; Energy Transfer; Plasmon Absorption; Super Quenching

\section{Introduction}

Gold nanoparticles are among the most extensively studied nanomaterials. They are known to be the most stable metal nanoparticles $[1,2]$. Numerous studies have been reported on the synthesis, property and application development of gold clusters, colloids, and nanoparticles [3-6]. Gold nanoparticles exhibit many unique and interesting physical and optical properties such as intense surface plasmon resonance (SPR) absorption, surface enhanced Raman scattering (SERS), nonlinear optical (NLO) properties, and quantized charging effect [7-9]. AuNPs are recognized as the building blocks for many nanoscale materials and devices including conductive and optical hybrid composites and promote their applications in diverse fields $[1-4,10]$. The surface plasmon resonance of gold nanoparticles is an optical property that holds promising potential in biosensing, molecular imaging, and photothermal treatment of diseases. Taton et al. demonstrated the use of gold nanoparticles as an optical probe to detect DNA molecules based on the color change of individu-

"Corresponding author. ally scattered nanoparticles and the complementary DNAhybridized nanoparticle aggregates [11].

Besides applications in optoelectronics and biomedicine, AuNP is known to have a superior quenching efficiency in a wider range of wavelengths compared to the organic quenchers [12-22]. AuNPs having unique size dependent SPR, its absorption band can be tuned easily and precisely by changing its shape and size to increase the overlap of its absorption band with the fluorescence band of a chosen fluorophore. This results in an efficient transfer of energy from the excited fluorophore to the gold nanoparticle surface leading to the quenching of the fluorescence of the fluorophore. To peep into the mechanistic aspect of the energy transfer process, two propositions are put forward; Förster resonance energy transfer (FRET) and nanoparticle surface energy transfer (NSET). In FRET the excitation energy is transferred to the acceptor dipole through induced dipole mechanism, while in NSET the dipolar interactions occur due to the presence of free conduction band electrons in the metal nanoparticle that provides dipole vectors on the surface of the metal ready to accept energy from the donor [18- 
28]. Apart from the conceptual difference, the major difference between FRET and NSET from the applicational point of view remains in the fact that while the efficiency of the former process depends on the inverse sixth power of the donor-acceptor distance $\left(1 / \mathrm{r}^{6}\right)$, for the latter the dependence lies on inverse fourth power $\left(1 / \mathrm{r}^{4}\right)$. Anyway, the high sensitivity of the energy transfer process on the donor-acceptor distance makes both FRET and NSET as "spectroscopic rulers" for measurement of intramolecular or intermolecular distances. This has provided physicists, chemists and biologists a powerful and versatile tool for studying the structure and dynamics of small or large molecules in condensed phases [18-28].

Keeping in mind the importance of search for newer donor-acceptor pairs for an efficient energy transfer, here we have explored quenching of the fluorescence of a well-known laser dye from the coumarin family, namely coumarin 153 (C153), by gold nanoparticles (AuNPs). Apart from being a laser dye, because of its solvent sensitive spectral behavior, C153 is exploited for deciphering various aspects including exploration of the properties of the different domains of microheterogeneous environments and solvation dynamics [29-32]. Considering a good overlap between the fluorescence spectrum of C153 emitting at $\sim 550 \mathrm{~nm}$ in water [29] and the absorption spectra of AuNPs, the C153-AuNP pair has been chosen for a plausible energy transfer from the donor (C153) to the acceptor (AuNPs). Since AuNPs have size dependent absorption bands with high extinction coefficients, we were expecting a super-efficient energy transfer system demonstrating a highly effective tunable quencher. We were also interested in deciphering the impact of the dimension of the gold nanoparticles on the efficiency of quenching. Hence, prior to probing of the quenching due to the energy transfer from the fluorophore to the AuNPs, we intended to synthesize the metal nanoparticles and standardize the method of synthesis. Since the optical behavior of AuNPs depend very much on their size, numerous research works of AuNPs are emphasized on controlling the particle size and morphology [33-35]. Attempts are also there to develop stabilizing methods with different functional ligands in solution [36]. Typically, the wet chemical synthesis of gold colloids is carried out in the presence of stabilizing agents, often citrate, phosphates or thiols, which bind to the atoms exposed at the surface of the nanoparticles. AuNPs can be synthesized with high degrees of precision for diameters between $2-300 \mathrm{~nm}$. As their diameters increase, their plasmon band shifts toward red making them highly tunable absorbing agents [37]. The simplest and by far the most commonly used method for preparation of gold sols is the reduction of aqueous $\mathrm{HAuCl}_{4}$ by sodium citrate at the boiling point [38,39]. Considering the important applications of AuNPs in diverse fields, a simple controlled synthesis of the nanoparticles under mild condition poses a challenge.

Our specific objective in this respect was to develop a simple strategy for the controlled synthesis as well as easy determination of the particle diameter of the synthesized gold nanoparticles. We have dealt with the synthesis of AuNPs by the citrate reduction of $\mathrm{HAuCl}_{4}$ as introduced by Frens, to produce gold nanoparticles within $15 \mathrm{~nm}-60 \mathrm{~nm}$ diameter [34,38] and have standardized this synthetic method with the help of TEM and spectrophotometric measurements. We have found correlations between the composition of the reactants in the reaction mixture with the maximum of the plasmon absorption $\left(\lambda_{\max }\right)$ as well as the diameter of the AuNPs produced. This allows one to dictate the dimension of the synthesized gold nanoparticles directly from the composition of the reactants and this can be confirmed easily from UV-vis spectrophotometry.

The outline of the present work thus remains two fold. Firstly to develop an effective strategy for the synthesis of gold nanoparticles of desired dimension controlling the composition of the reaction mixture and secondly to use these AuNPs as super-efficient quenchers for suitable fluorophores.

The impetus of the work lies in providing a newer and simple system to be exploited for developing biosensor with high degree of sensitivity since AuNPs can be tagged to biomacromolecules. The high quenching efficiency of gold nanoparticles has found potential applications in the development of fluorescence-based assays for DNA, RNA and protein markers [40]. Gold nanoparticles are also observed to quench the conjugated polymers superefficiently which has its applications in the polymerbased biosensing at the subpicomolar analyte concentration [14]. The high extinction coefficient near the plasmon resonance frequency enhances the efficiency of the gold nanoparticles towards quenching of the fluorescence of the matched fluorophores [16]. This super quenching property of AuNPs allows them to be developed as gold nanoparticle-based miniaturized energy transfer probe and employed in "turn on" and "turn off" fluorescence approaches for the sensitive and selective detection of $\mathrm{Hg}^{2+}, \mathrm{Pb}^{2+}$, and $\mathrm{Cu}^{2+}$ ions $[41,42]$.

\section{Experimental Details}

Coumarin 153 and hydrogen tetrachloroaurate trihydrate $\left(\mathrm{HAuCl}_{4}\right)$ purchased from Sigma-Aldrich (USA) and trisodium citrate dihydrate from Merck (India) respectively are used without further purification. Triply distilled water has been used to prepare the aqueous solutions wherever required.

Gold nanoparticles of different sizes have been synthesized following the citrate reduction of $\mathrm{HAuCl}_{4}$ as introduced by Frens [38,39] (Scheme 1). AuNPs of vari- 


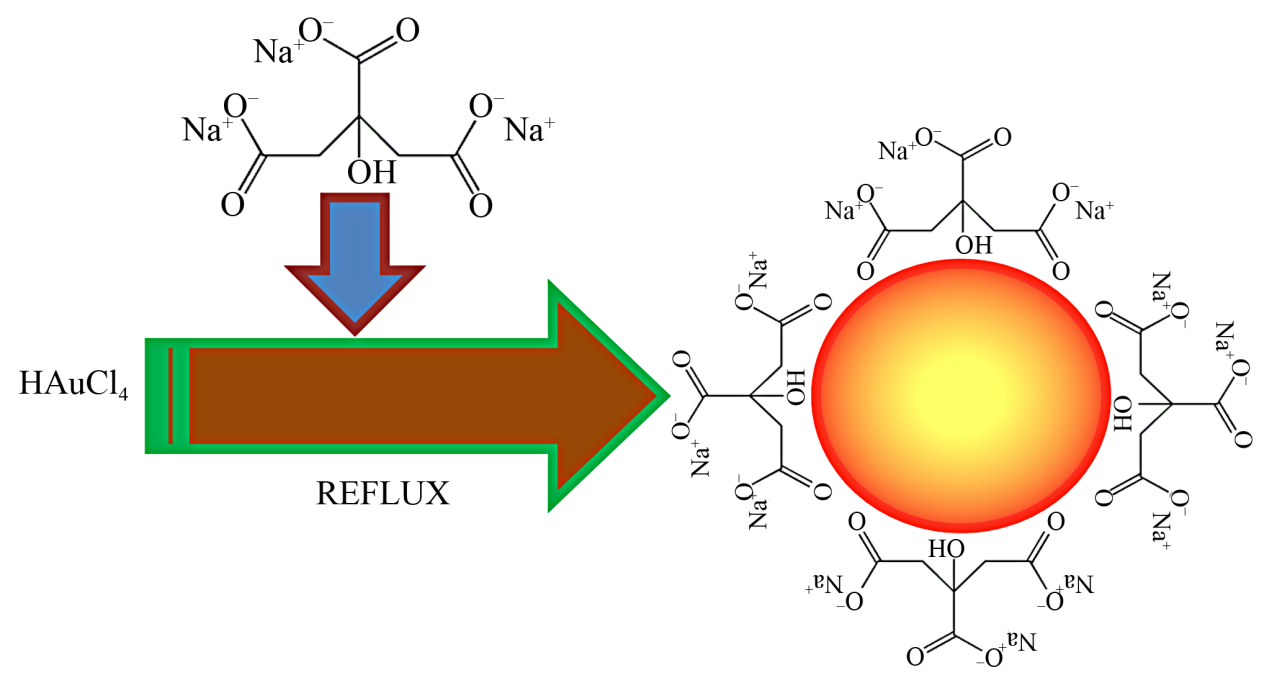

Scheme 1. Schematic presentation of preparation of citrate stabilized AuNP in water.

ous particle diameters have been synthesized by controlling the fractional concentration of the reducing agent in the reaction mixture [39].

Concentration of the synthesized AuNPs is calculated from the concentration of gold salt in the reaction mixture following the procedure reported by El-Sayed et al. [43] considering the reduction to be quantitative. The aggregation number is calculated using the particle radius estimated from the TEM images and taking the atomic radius of gold to be 144 picometer. The molar extinction coefficient of the synthesized AuNPs is then calculated from the aggregation number, concentration of gold salt in the reaction mixture and the absorbance of the respective AuNPs.

Room temperature absorption and steady state fluorescence measurements are performed using a Shimadzu UV-2450 spectrophotometer and a Spex fluorolog-2 spectrofluorometer equipped with DM3000F software respectively. Fluorescence lifetimes are determined from time-resolved intensity decays by the method of timecorrelated single-photon counting (TCSPC) using a nanosecond diode excitation source at $403 \mathrm{~nm}$ (IBH, UK, nanoLED-07) and TBX-04 as the detector. The instrument response time is $\sim 1$ ns. The decays are deconvoluted using IBH DAS-6 decay analysis software. Quality of the fits is judged by the reduced $\chi^{2}$ criterion and the randomness of the fitted function to the raw data. Biexponential decay profiles are described by $I(t)=a_{1}$ $\exp \left(-t / \tau_{1}\right)+a_{2} \exp \left(-t / \tau_{2}\right)$, where $\tau_{1}$ and $\tau_{2}$ are the decay times and $a_{1}$ and $a_{2}$ are their relative amplitudes. The amplitude averaged fluorescence lifetime $(\tau)$ has been defined as

$$
\tau=\left(a_{1} \tau_{1}+a_{2} \tau_{2}\right) /\left(a_{1}+a_{2}\right)
$$

All the experiments are performed at ambient temperature $\left(27^{\circ} \mathrm{C}\right)$ with air-equilibrated solutions.

\section{Results and Discussion}

\subsection{Standardization of Synthesis of Gold Nanoparticles of Desired Dimension in the Range 15 nm - 60 nm}

The absorption spectra of the synthesized gold nanoparticles of four different dimensions are depicted in Figure 1. The figure reveals the characteristic surface plasmon bands. The high extinction coefficients of the AuNPs are manifested in the intense absorption bands. While varying the fractional concentration of citrate in the reaction mixture from 0.4 to 0.728 there was a hypsochromic shift in the absorption maximum $\left(\lambda_{\max }\right)$ of the synthesized gold nanoparticles from $535 \mathrm{~nm}-520 \mathrm{~nm}$. We have tried to find if there is any correlation between the $\lambda_{\max }$ and the particle size. For the purpose of getting the dimensions of the AuNPs, we take help of TEM. The morphology of the synthesized gold nanoparticles from the TEM images reveals spherical gold nanoparticles. For four different gold nanoparticles of varying sizes the diameters fall in the range of $16 \mathrm{~nm}$ to $59 \mathrm{~nm}$ (Table 1). It has already been reported by Frens, although qualitatively, that an alteration of concentration ratio of sodium citrate to tetrachloroaurate, used for the reduction reaction, changes the particle size [38]. Our results corroborate it quantitatively (vide supra).

To develop a possible strategy to assess the diameter of the synthesized AuNPs directly from the composition of the reaction mixture through a relation, we looked for a series of variations between different parameters related to the aspect (Figure 2). The variation of diameter of the AuNPs as directly measured from TEM was noticed as a function of $\lambda_{\max }$ (Figure 2(a)). Then the variation of $\lambda_{\max }$ was studied as a function of the fractional concentration of the reductant (citrate) in the reaction mixture (Figure 2(b)). Finally the correlation for the 


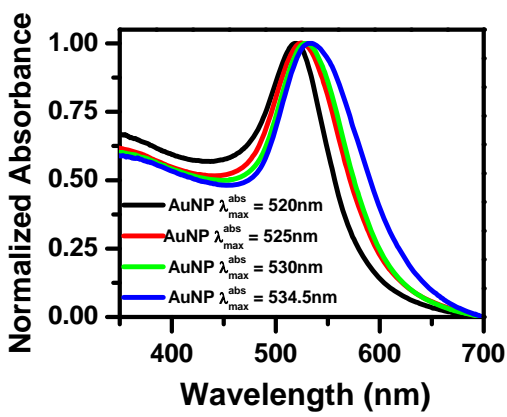

Figure 1. Absorption spectra of the synthesized gold nanoparticles.

Table 1. Absorption maxima and diameters of synthesized AuNPs.

\begin{tabular}{cc}
\hline$\lambda_{\max }(\mathrm{nm})$ & Diameter $(\mathrm{nm})$ \\
\hline 520 & 16 \\
525 & 30 \\
530 & 46 \\
534.5 & 59 \\
\hline
\end{tabular}

variation of particle diameter as a function of the fractional concentration of citrate was determined (Figure 2(c)). Interestingly, the last correlation was same as that calculated from the first two correlations. This agreement emphasizes the reliability of our strategy and enables one to assess the diameter of the AuNPs to be synthesized directly from the knowledge of the fractional concentration of the citrate in the reaction mixture while adopting Frens method.

Figure 2(a) depicts the plot of particle diameter of the AuNPs measured directly from TEM, as a function of $\lambda_{\max }$. This plot reveals a linear variation with a very good correlation factor $(R=0.999)$. This emphasizes on the fact that one can rely on either of the two factors almost equally. This is in tune with the proposition of Mie theory [44]. This plot, thus, provides an easier way to estimate the size of the nanoparticles from the knowledge of the $\lambda_{\max }$ of the synthesized AuNPs without using the costlier, and often not available, TEM. Considering the fact that composition of the reaction mixture during the synthesis of the AuNPs plays as the controlling factor for the size of the synthesized gold nanoparticles and that $\lambda_{\max }$ depends on the size of the nanoparticles, we looked for finding a correlation between $\lambda_{\max }$ and fractional concentration of the reductant (citrate) in the reaction mixture. Fractional concentration $(F C)$ of the citrate salt in the reaction mixture is defined as $F C=[$ Citrate $] /\left([\right.$ Citrate $\left.]+\left[\mathrm{HAuCl}_{4}\right]\right)$. Figure 2(b) depicts a linear variation between these two factors for the synthesized AuNPs giving, again, a very good correlation $(R=0.993)$. From this plot one gets a direct correlation between the maximum of the plasmon absorption band and the fractional concentration of the reductant.
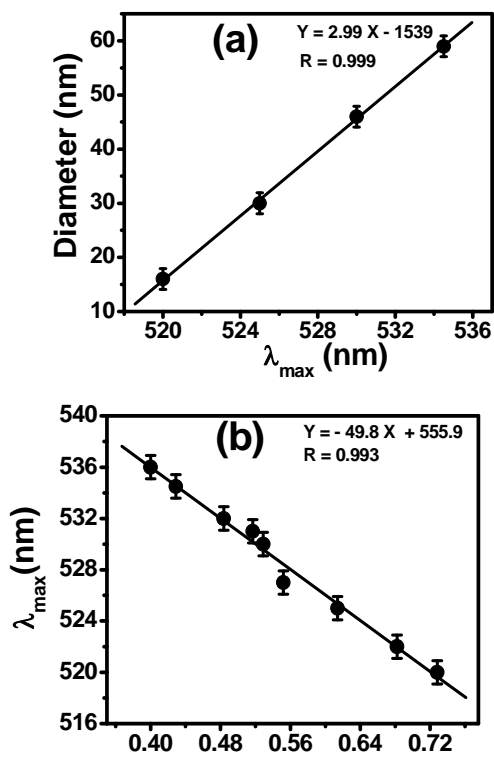

Fractional concentration of citrate

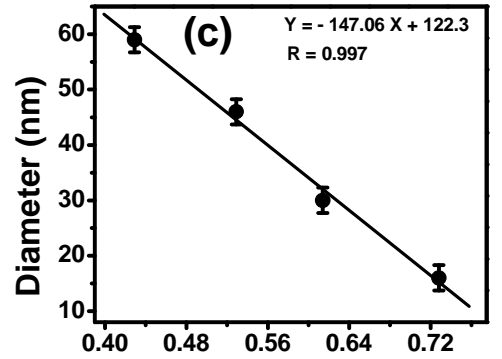

Fractional concentration of citrate

Figure 2. (a) Plot of diameter of the gold nanoparticles measured from TEM against absorption maxima of AuNPs; (b) Plot of absorption maxima of the AuNPs with the fractional concentration of citrate in the reaction mixture; (c) Plot of diameter of the AuNPs with the fractional concentration of citrate in the reaction mixture.

To make the citrate reduction method programmable in the sense that one can assess the dimension of the synthesized AuNPs simply from the composition of the reaction mixture, we intended to obtain a correlation between the diameter of the synthesized AuNPs and the fractional concentration of citrate in the reaction mixture. Figure 2(c) depicts the calibration plot between these two parameters with a good correlation factor once again ( $R=0.997)$. This calibration plot enables one to synthesize gold nanoparticles of a desired dimension, at least within the range $15 \mathrm{~nm}-60 \mathrm{~nm}$, directly by controlling the concentration of citrate relative to $\mathrm{HAuCl}_{4}$ in the reaction mixture. It is encouraging to note that the slope of Figure 2(c) agrees well with the product of the slopes of Figures 2(a) and (b). The cross correlation between the three parameters dealt with is thus established and enables one to exploit the standardized method of synthesis of AuNPs to assess the dimension of the nanoparticle from the fractional concentration of the citrate in the 
mixture. Figure 2(c) projects the correlation between the diameter of the synthesized AuNP and composition of citrate in the mixture as:

$$
\begin{aligned}
& \text { Diameter of AuNP } \\
= & -147.06 \times(\text { fractional concentration of citrate }) \\
& +122.3
\end{aligned}
$$

Standardization of the citrate reduction method of synthesis of gold nanoparticles, based exclusively on the instrumental support, makes the method very friendly to use.

To make the story short, let us elucidate the steps involved during the development of the strategy. 1) First we vary the concentration of the citrate keeping the concentration of the hydrogen tetrachloroaurate trihydrate $\left(\mathrm{HAuCl}_{4}\right)$ constant to synthesize AuNPs of different sizes; 2) Then we measure the diameters of the synthesized gold nanoparticles from transmission electron microscopy (TEM); 3) We collect the absorption spectra and get the plasmonic absorption maxima $\left(\lambda_{\max }\right)$ for the synthesized gold nanoparticles; 4) Now we plot these $\lambda_{\max }$ against the measured diameters of the AuNPs and get a correlation between the two parameters; 5) We also calculate the fractional concentration $(F C)$ of the citrate in the reaction mixture as

$F C=[$ Citrate $] /\left([\right.$ Citrate $\left.\left.]+\left[\mathrm{HAuCl}_{4}\right]\right) ; 6\right) \lambda_{\max }$ is then plotted against calculated $F C$ of citrate for all the synthesized AuNPs to get a correlation; 7) The diameters of the nanoparticles measured from the TEM are then plotted against $F C s$ of citrate used for the synthesis of nanoparticles of different dimensions to get the final correlation between the diameter of the AuNPs and the $F C$ of the citrate in the reaction mixture. The final equation that is developed through this strategy is given above (Equation (2)). Following this relation, one can simply set a definite composition of the reactants in the reaction mixture and calculate the expected diameter of AuNP to be synthesized from the $F C$ value of the reaction mixture. After the actual synthesis, one can confirm the diameter simply from spectrophotometry.

\subsection{Superquenching of Coumarin 153 by Gold Nanoparticles}

After synthesizing the AuNPs of desired dimensions (within the range $15 \mathrm{~nm}-60 \mathrm{~nm}$ ) we proceeded to study the effect of these metal nanoparticles on the fluorescence of coumarin 153. In aqueous medium photoexcitation of $\mathrm{C} 153\left(\sim 1 \times 10^{-5} \mathrm{~mol} \cdot \mathrm{dm}^{-3}\right)$ at its lowest energy absorption band $(430 \mathrm{~nm})$ yields a broad and unstructured fluorescence peaking at around $550 \mathrm{~nm}$ [29]. Successive addition of picomolar concentrations of AuNPs in this solution leads to a remarkable quenching of the emission of the fluorophore. Repetition of the steady state fluorescence quenching of $\mathrm{C} 153$ using gold nanoparticles of four different dimensions reflects that the degree of fluorescence quenching increases radically with an increase in the size of the gold nanoparticles.

The quenching phenomenon could result either from static or dynamic interactions between the dye and the nanoparticles. To confirm the character of the quenching process we have performed the time-resolved fluorescence decay analyses $[19,23]$. In aqueous solution, fluorescence of $\mathrm{C} 153$ at $550 \mathrm{~nm}$ displays a bi-exponential decay giving an average lifetime of $2 \pm 0.2 \mathrm{~ns}$ [29]. Upon successive addition of AuNPs to the solution, a gradual decrease in the fluorescence lifetime of $\mathrm{C} 153$ is observed. Figure 3 depicts a representative set of the decay profiles of $\mathrm{C} 153$ in the presence of varying concentrations of AuNP of a particle diameter, namely, $30 \mathrm{~nm}$. A marked lowering in the donor lifetime confirms the quenching process to be dynamic $[23,45]$. For a definite concentration of the quencher (AuNPs), the fluorescence lifetime of $\mathrm{C} 153$ is found to decrease more with an increase in size of the quencher nanoparticles, consistent with the steady state observations.

Stern-Volmer relation is observed to be followed for the quenching of the fluorescence lifetime of the donor with the addition of the AuNPs (Figure 4).

$$
\tau_{0} / \tau=1+K_{S V}[\mathrm{AuNP}]
$$

$\tau_{0}$ and $\tau$ are the fluorescence lifetimes of $\mathrm{C} 153$ in the absence and presence of the AuNPs respectively. Slopes of the plots depicted in Figure 4 give the Stern-Volmer constants and they come to be in the range $10^{7}-10^{9}$ $\mathrm{mol}^{-1} \cdot \mathrm{dm}^{3}$. As expected, the $K_{S V}$ increases with an increase in the dimension of the AuNPs with values $4.5 \times$ $10^{7}, 2.76 \times 10^{8}, 1.27 \times 10^{9}$ and $6.37 \times 10^{9} \mathrm{~mol}^{-1} \cdot \mathrm{dm}^{3}$ for $16,30,46$ and $59 \mathrm{~nm}$ particle respectively.

It is interesting to note that the values of the SternVolmer constants are orders of magnitude higher compared to the same for normal diffusion controlled quenching processes [10]. This substantiates the super-efficient

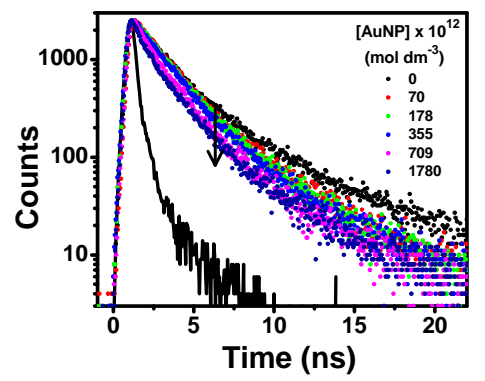

Figure 3. Time-resolved fluorescence decays of C153 $(\sim 1 \times$ $\left.10^{-5} \mathrm{~mol} \cdot \mathrm{dm}^{-3}\right)$ in water in the presence of varying concentrations of AuNP of diameter $30 \mathrm{~nm}$. The concentrations of AuNPs are provided in the legends. The line profile in black represents the instrument response function. $\lambda_{\mathrm{exc}}=403 \mathrm{~nm}$, $\lambda_{\mathrm{em}}=550 \mathrm{~nm}$. 

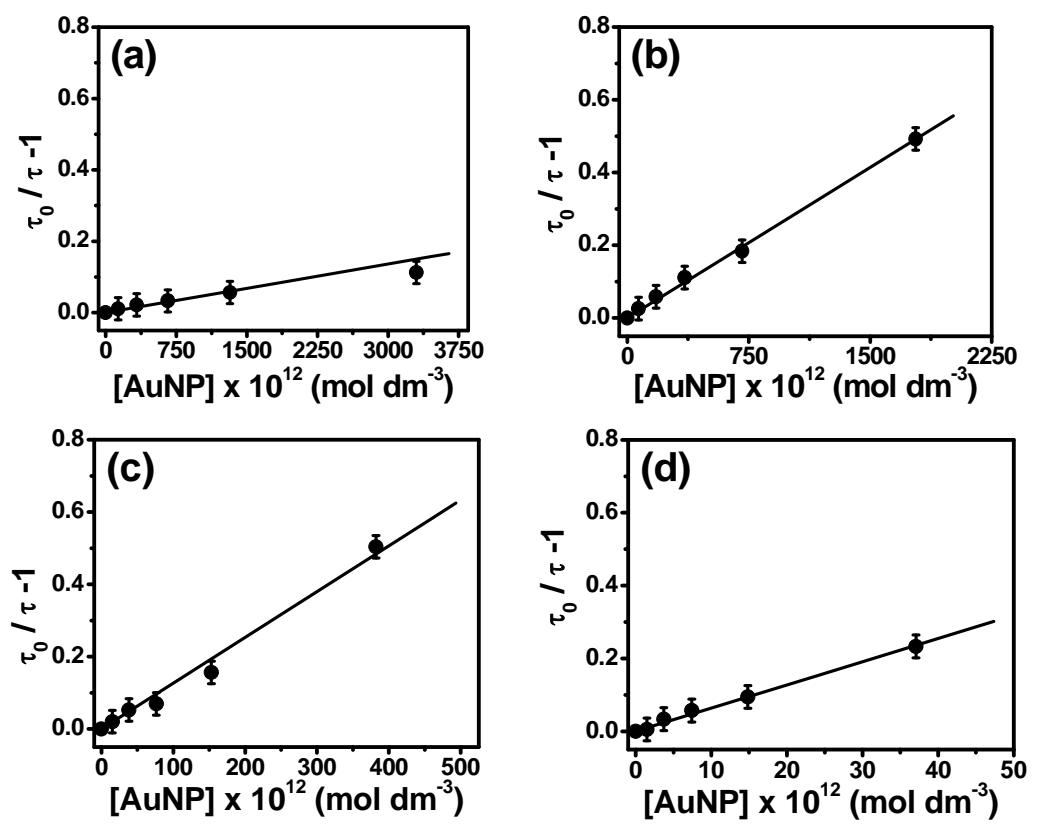

Figure 4. Stern-Volmer plots for the quenching of the fluorescence lifetime of C153 upon addition of AuNPs of particle diameters (a) $16 \mathrm{~nm}$; (b) $30 \mathrm{~nm}$; (c) $46 \mathrm{~nm}$; and (d) $59 \mathrm{~nm}$. The ordinate scale has been kept same for a better appreciation of the relative quenching effect.

quenching of the donor fluorescence by the gold nanoparticles. The very high $K_{S V}$ values ascribe the quenching to be an efficient non-radiative energy transfer from C153 to the AuNPs $[10,46,47]$. Since the dimensions of the AuNPs for the present study $(15-59 \mathrm{~nm})$ are greater than $10 \mathrm{~nm}$, we see an efficient quenching instead of an plasmonic near-field enhancement which is reported for smaller AuNPs ( $\leq 10 \mathrm{~nm})[48,49]$.

The energy transfer efficiencies $(E)$ have been measured using Equation (4) from the lifetime data. $\tau$ and $\tau_{0}$ are the lifetimes of the donor in the presence and absence of the acceptor respectively.

$$
E=1-\left(\tau / \tau_{0}\right)
$$

The dependence of the critical donor-acceptor distance $\left(R_{0}\right)$ when energy transfer efficiency is $50 \%$, on the spectral overlap (Figure 5) for a particular donor-acceptor pair is expressed as

$$
R=8.8 \times 10^{23}\left[\kappa^{2} \cdot n^{-4} \cdot \Phi_{D} \cdot J(\lambda)\right]
$$

where $\kappa^{2}$ represents the relative orientation of the donor to the acceptor molecule, $n$ is the refractive index of the medium (1.333 for water), $\Phi_{D}$ is the quantum yield of the donor in the absence of the acceptor, taken as 0.12 from the literature [50,51] and $J(\lambda)$ is the overlap integral of the fluorescence emission spectrum of the donor and the absorption spectrum of the acceptor in units of $\mathrm{mol}^{-1} \cdot \mathrm{dm}^{3} \cdot \mathrm{cm}^{3}$. Considering a random rotational diffusion for the small molecules, $\kappa^{2}$ is taken to be $2 / 3$ [23]. The overlap integral $J(\lambda)$ has been calculated from the

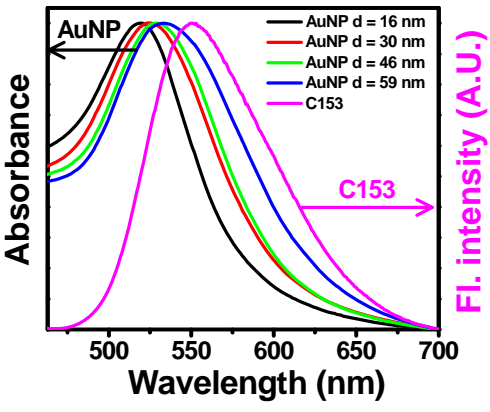

Figure 5. Overlap between the fluorescence spectrum of C153 (donor) and the absorption spectra of AuNPs (acceptor).

numerical integration method using the following relation [23].

$$
J(\lambda)=\frac{\int_{0}^{\infty} F(\lambda) \varepsilon_{A}(\lambda) \lambda^{4} \mathrm{~d} \lambda}{\int_{0}^{\infty} F(\lambda) \mathrm{d} \lambda}
$$

where $F(\lambda)$ is the fluorescence intensity of the donor in the wavelength range $\lambda$ to $(\lambda+\Delta \lambda)$ with the total intensity normalized to unity, $\varepsilon_{A}(\lambda)$ is the molar extinction coefficient of the acceptor at wavelength $\lambda$ expressed in $\mathrm{mol}^{-1} \cdot \mathrm{dm}^{3} \cdot \mathrm{cm}^{-1}$. The overlap integrals $J(\lambda)$ for the present donor-acceptor (C153-AuNP) are estimated to be $2.54 \times$ $10^{-11}, 3.81 \times 10^{-11}, 5.13 \times 10^{-11}$ and $7.03 \times 10^{-11}$ $\mathrm{mol}^{-1} \cdot \mathrm{dm}^{3} \mathrm{~cm}^{3}$ for $16 \mathrm{~nm}, 30 \mathrm{~nm}, 46 \mathrm{~nm}$ and $59 \mathrm{~nm}$ AuNPs respectively. Using Equation (5), the corresponding $\mathrm{R}_{0} \mathrm{~s}$ have been evaluated to be $91.1 \AA, 97.5 \AA, 102.5$ $\AA$ and $108 \AA$ [52]. This infers that the larger gold nanopar- 
ticles are capable of quenching the fluorescence of C153 from a longer distance, that is, the energy transfer efficiency is higher for bigger AuNPs. This is ascribed to the much enhanced extinction coefficient, and a greater overlap between the fluorescence spectrum of C153 and AuNPs as the dimension of the latter is increased (Figure 5).

Using the experimental values of $\mathrm{E}$ and the $R_{0} s$ for the AuNPs of various diameters, for both FRET and NSET the general relation correlating $E$ with $r$ (Equation (7)) could be fitted well (Figure 6).

$$
E=\frac{1}{1+\left(r / R_{0}\right)^{n}},
$$

with $n=6$ for FRET and $n=4$ for NSET

For NSET, however, $R_{0}$ can be calculated independently using Persson-Lang model (Equation (8)) [53].

$$
R_{0}=\left(\frac{0.225 c^{3} \Phi_{D}}{\omega_{D}^{2} \omega_{F} k_{F}}\right)^{1 / 4}
$$

where $c$ is the speed of light, $\Phi_{D}$ is the quantum yield of the donor in the absence of the acceptor, $\omega_{D}$ is the angular frequency for the donor $\left(3.43 \times 10^{15} \mathrm{~s}^{-1}\right), \omega_{F}$ is the angular frequency of the bulk gold $\left(8.4 \times 10^{15} \mathrm{~s}^{-1}\right)[19$, 20,22] and $k_{F}$ is the Fermi wave vector for bulk gold (1.2 $\left.\times 10^{8} \mathrm{~cm}^{-1}\right)[19,20,22]$. From these standard data we get $R_{0}$ to be $49.8 \AA$. The simulated curve using $n=4$ and this value of $R_{0}$ is also incorporated in Figure 6(b). Although not conclusive, the well off and a much lower value of this $R_{0}$ obtained from Persson-Lang model suggests that in the present energy transfer case, the operating mechanism is FRET and not NSET.

\section{Conclusion}

The citrate reduction method of synthesis of gold nanoparticles is standardized. Using the developed correlation one can assess the dimension of the AuNP to be synthesized, in advance, from the composition of the reaction mixture and the diameter of the synthesized ones can be confirmed simply from spectrophotometry. This essentially makes the use of costly TEM unnecessary, at least for the primary purposes. Furthermore, a highly efficient energy transfer from the laser dye coumarin 153 to the synthesized gold nanoparticles (AuNP) of varying dimensions has been demonstrated. The very high values of the Stern-Volmer constants $\left(10^{7}-10^{9} \mathrm{~mol}^{-1} \cdot \mathrm{dm}^{3}\right) \mathrm{de}-$ termined from the time-resolved quenching studies in the presence of AuNPs of varying sizes are orders of magnitude higher than those for the normal photochemical quenching processes. This is ascribed to an efficient energy transfer from C153 to AuNPs. A large enhancement in the extinction coefficient and an increase in the spectral overlap between the plasmonic absorption band of
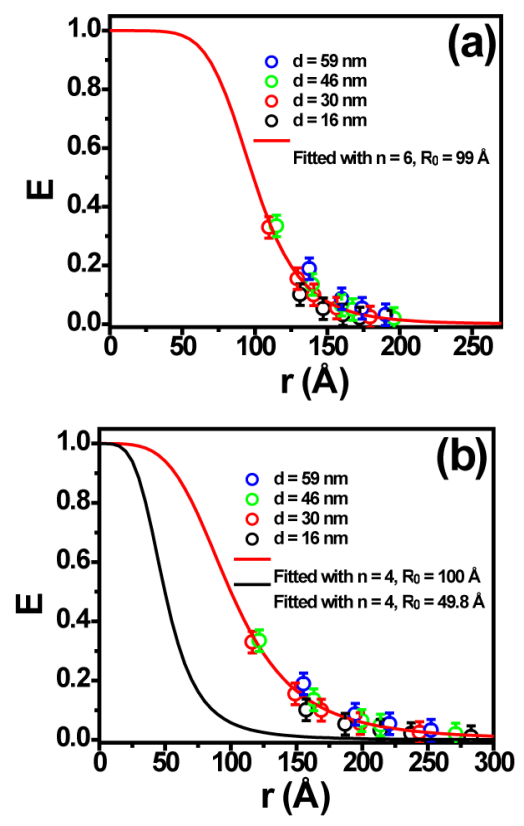

Figure 6. Experimentally determined energy transfer efficiency plotted against the distance between dye and AuNPs of different sizes estimated from (a) FRET and (b) NSET models. The AuNPs of different diameters have been designated in the legends. The lines are the theoretical fits taking quenching data for all the particles together following the respective models. The black line in (b) represents simulation with $R_{0}=49.8 \AA$ (calculated from Persson-Lang model).

the AuNPs and the fluorescence spectrum of C153 with an increase in the size of the nanoparticles are held responsible for the enhanced energy transfer efficiency. The importance of the work lies in providing a new and simple system to be exploited for developing biosensors with high degree of sensitivity when the AuNPs are tagged to the biomacromolecules.

\section{Acknowledgements}

Financial support from the Department of Science and Technology, Govt. of India, is gratefully acknowledged.

\section{REFERENCES}

[1] A. Borriello, P. Agoretti, A. Cassinese, P. D’Angelo, G. T. Mohanraj and L. Sanguigno, "Electrical Bistability in Conductive Hybrid Composites of Doped Polyaniline Nanofibers-Gold Nanoparticles Capped with Dodecane Thiol," Journal of Nanoscience and Nanotechnology, Vol. 9, No. 11, 2009, pp. 6307-6314. doi:10.1166/jnn.2009.1470

[2] C.-W. Hu, Y. Huang and R. C.-C. Tsiang, "Thermal and Spectroscopic Properties of Polystyrene/Gold Nanocomposite Containing Well-Dispersed Gold Nanoparticles," Journal of Nanoscience and Nanotechnology, Vol. 9, No. 5, 2009, pp. 3084-3091. doi:10.1166/jnn.2009.003 
[3] M.-C. Daniel and D. Astruc, "Gold Nanoparticles: Assembly, Supramolecular Chemistry, Quantum-Size-Related Properties, and Applications toward Biology, Catalysis, and Nanotechnology," Chemical Reviews, Vol. 104, No. 1, 2002, pp. 293-346. doi:10.1021/cr030698+

[4] C. Burda, X. Chen, R. Narayanan and M. A. El-Sayed, "Chemistry and Properties of Nanocrystals of Different Shapes," Chemical Reviews, Vol. 105, No. 4, 2005, pp. 1025-1102. doi:10.1021/cr030063a

[5] E. Katz and I. Willner, "Integrated Nanoparticle-Biomolecule Hybrid Systems: Synthesis, Properties, and Applications," Angewandte Chemie International Edition, Vol. 43, No. 45, 2004, pp. 6042-6108. doi:10.1002/anie.200400651

[6] M. J. Kogan, N. G. Bastus, R. Amigo, D. Grillo-Bosch, E. Araya, E. Araya, A. Turiel, A. Labarta, E. Giralt and V. F. Puntes, "Nanoparticle-Mediated Local and Remote Manipulation of Protein Aggregation," Nano Letters, Vol. 6, No. 1, 2006, pp. 110-115. doi:10.1021/n10516862

[7] T. Klar, M. Perner, S. Grosse, G. von Plessen, W. Spirkl and J. Feldmann, "Surface-Plasmon Resonances in Single Metallic Nanoparticle," Physical Review Letters, Vol. 80, No. 19, 1998, pp. 4249-4252. doi:10.1103/PhysRevLett.80.4249

[8] Y. W. C. Cao, R. Jin and C. A. Mirkin, "Nanoparticles with Raman Spectroscopic Fingerprints for DNA and RNA Detection," Science, Vol. 297, No. 5586, 2002, pp. 1536-1540. doi:10.1126/science.297.5586.1536

[9] R. D. Averitt, S. L.Westcott and N. J. Halas, "Linear Optical Properties of Gold Nanoshells," Optics InfoBase: Journal of the Optical Society of America B, Vol. 16, No. 10, 1999, pp. 1824-1832. doi:10.1364/JOSAB.16.001824

[10] V. G. Praig, H. McIlwee, C. L. Schauer, R. Boukherroub and S. Szunerits, "Localized Surface Plasmon Resonance of Gold Nanoparticle-Modified Chitosan Films for HeavyMetal Ions Sensin," Journal of Nanoscience and Nanotechnology, Vol. 9, No. 1, 2009, pp. 350-357. doi:10.1166/jnn.2009.J064

[11] T. A. Taton, G. Lu and C. A. Mirkin, "Two-Color Labeling of Oligonucleotide Arrays via Size-Selective Scattering of Nanoparticle Probes," Journal of the American Chemical Society, Vol. 123, No. 21, 2001, pp. 5164-5165. doi:10.1021/ja0102639

[12] E. Dulkeith, A. C. Morteani, T. Niedereichholz, T. A. Klar, J. Feldmann, S. A. Levi, F. C. J. M. van Veggel, D. N. Reinhoudt, M. Moller and D. I. Gittins, "Fluorescence Quenching of Dye Molecules near Gold Nanoparticles: Radiative and Nonradiative Effects," Physical Review Letters, Vol. 89, No. 20, 2002, Article ID: 203002/1-4. doi:10.1103/PhysRevLett.89.203002

[13] B. Dubertret, M. Calame and A. J. Libchaber, "Single-Mismatch Detection Using Gold-Quenched Fluorescent Oligonucleotides," Nature Biotechnology, Vol. 19, No. 4, 2001, pp. 365-370. doi:10.1038/86762

[14] C. Fan, S. Wang, J. W. Hong, G. C. Bazan, K. W. Plaxco and A. J. Heeger, "Beyond Superquenching: Hyper-Efficient Energy Transfer from Conjugated Polymers to Gold Nanoparticles," Proceedings of the National Academy of Sciences, Vol. 100, No. 11, 2003, pp. 6297-6301. doi:10.1073/pnas.1132025100

[15] Q. Xu, J. Liu, Z. He and S. Yang, "Superquenching Acridinium Ester Chemiluminescence by Gold Nanoparticles for DNA Detection," Chemical Communications, Vol. 46. No. 46, 2010, pp. 8800-8802. doi: $10.1039 / \mathrm{c} 0 \mathrm{cc} 03349 \mathrm{a}$

[16] J. Griffin, A. K. Singh, D. Senapati, P. Rhodes, K. Mitchell, B. Robinson, E. Yu and P. C. Ray, "Size- and Distance-Dependent Nanoparticle Surface Energy Transfer (NSET) Method for Selective Sensing of Hepatitis C Virus RNA," Chemistry-A European Journal, Vol. 15, No. 2, 2009, pp. 342-351. doi:10.1002/chem.200801812

[17] M. Li, S. K. Cushing, Q. Wang, X. Shi, L. A. Hornak, Z. Hong and N. Wu, "Size-Dependent Energy Transfer between CdSe/ZnS Quantum Dots and Gold Nanoparticles," The Journal of Physical Chemistry Letters, Vol. 2, No. 17, 2011, pp. 2125-2129. doi:10.1021/jz201002g

[18] C. S. Yun, A. Javier, T. Jennings, M. Fisher, S. Hira, S. Peterson, B. Hopkins, N. O. Reich and G. F. Strouse, "Nanometal Surface Energy Transfer in Optical Rulers, Breaking the FRET Barrier," Journal of the American Chemical Society, Vol. 127, No. 9, 2005, pp. 3115-3119. doi:10.1021/ja043940i

[19] T. L. Jennings, M. P. Singh and G. F. Strouse, "Fluorescent Lifetime Quenching Near d = $1.5 \mathrm{~nm}$ Gold Nanoparticles: Probing NSET Validity," Journal of the American Chemical Society, Vol. 128, No. 16, 2006 pp. 5462-5467. doi: $10.1021 / \mathrm{ja} 0583665$

[20] T. L. Jennings, J. C. Schlatterer, M. P. Singh, N. L. Greenbaum and G. F. Strouse, "NSET Molecular Beacon Analysis of Hammerhead RNA Substrate Binding and Catalysis," Nano Letters, Vol. 6, No. 7, 2006, pp. 13181324. doi: $10.1021 / \mathrm{n} 1052458 \mathrm{a}$

[21] T. Pons, I. L. Medintz, K. E. Sapsford, S. Higashiya, A. F. Grimes, D. S. English and H. Mattoussi, "On the Quenching of Semiconductor Quantum Dot Photoluminescence by Proximal Gold Nanoparticles," Nano Letters, Vol. 7, No. 10, 2007, pp. 3157-3164. doi:10.1021/n1071729+

[22] T. Sen, S. Sadhu and A. Patra, "Surface Energy Transfer from Rhodamine 6G to Gold Nanoparticles: A Spectroscopic Ruler," Applied Physics Letters, Vol. 91, No. 4, 2007, Article ID: 043104/1-3. doi:10.1063/1.2762283

[23] J. R. Lakowicz, "Principles of Fluorescence Spectroscopy," 3rd Edition, Springer, New York, 2006. doi:10.1007/978-0-387-46312-4

[24] P. G. Wu and L. Brand, "Resonance Energy Transfer: Methods and Applications," Analytical Biochemistry, Vol. 218, No. 1, 1994, pp. 1-13. doi:10.1006/abio.1994.1134

[25] B. W. Van Der Meer, G. Coker III and S-Y. S. Chen, "Resonance Energy Transfer: Theory and Data," VCH, New York, 1994.

[26] P. R. Selvin, "Fluorescence Resonance Energy Transfer," Methods in Enzymology, Vol. 246. No. 1995, pp. 300334.

[27] D. Sarkar, P. Das and N. Chattopadhyay, "Fluorescence Resonance Energy Transfer: A Spectroscopic Ruler," ISRAPS Bulletin, Vol. 20, No. 2, 2008, pp. 31-41. 
[28] R. R. Chance, A. Prock and R. Silbey, "Molecular Fluorescence and Energy Transfer near Interfaces," Advances in Chemical Physics, Vol. 37, Wiley \& Sons, New York, 1978. doi:10.1002/9780470142561.ch1

[29] D. Seth, S. Sarkar and N. Sarkar, "Dynamics of Solvent and Rotational Relaxation of Coumarin 153 in a Room Temperature Ionic Liquid, 1-Butyl-3-methylimidazolium Octyl Sulfate, Forming Micellar Structure," Langmuir, Vol. 24, No. 14, 2008, pp. 7085-7091. doi:10.1021/la800813u

[30] Y. Kimura and N. Hirota, "Effect of Solvent Density and Species on Static and Dynamic Fluorescence Stokes Shifts of Coumarin 153," Journal of Chemical Physics, Vol. 111, No. 12, 1999, pp. 5474-5484. doi:10.1063/1.479808

[31] M. Maroncelli, "The Dynamics of Solvation in Polar Liquids," Journal of Molecular Liquids, Vol. 57, No. 1, 1993, pp. 1-37. doi:10.1016/0167-7322(93)80045-W

[32] J. E. Lewis and M. Maroncelli, "On the (Uninteresting) Dependence of the Absorption and Emission Transition Moments of Coumarin 153 on Solvent," Chemical Physics Letters, Vol. 282, No. 2, 1998, pp. 197-203. doi:10.1016/S0009-2614(97)01270-0

[33] A. Henglein and D. Meisel, "Radiolytic Control of the Size of Colloidal Gold Nanoparticles," Langmuir, Vol. 14, No. 26, 1998, pp. 7392-7396. doi:10.1021/la981278w

[34] N. R. Jana, L. Gearheart and C. J. Murphy, "Seeding Growth for Size Control of 5 - $40 \mathrm{~nm}$ Diameter Gold Nanoparticle," Langmuir, Vol. 17, No. 22, 2001, pp. 6782-6786. doi:10.1021/la0104323

[35] Y. G. Sun and Y. N. Xia,"Shape-Controlled Synthesis of Gold and Silver Nanoparticles," Science, Vol. 298, No. 5601, 2002, pp. 2176-2179. doi:10.1126/science.1077229

[36] A. C. Templeton, W. P. Wuelfing and R. W. Murray, "Monolayer-Protected Cluster Molecules," Accounts of Chemical Research, Vol. 33, No. 1, 2000, pp. 27-36. doi:10.1021/ar9602664

[37] D. A. Handley, "Methods for Synthesis of Colloidal Gold," In: M. A. Hayat, Ed., Colloidal Gold: Principles, Methods, and Applications, Vol. 1, Chapter 2, Academic Press, New York, 1989.

[38] G. Frens, "Controlled Nucleation for the Regulation of the Particle Size in Monodisperse Gold Suspension," $\mathrm{Na}$ ture Physics, Vol. 241, No. 1, 1973, pp. 20-22.

[39] J. Turkevich, P. C. Stevenson and J. Hillier, "The Formation of Colloidal Gold," The Journal of Physical Chemistry, Vol. 57, No. 7, 1953, pp. 670-673. doi:10.1021/j150508a015

[40] M. Swierczewska, S. Lee and X. Chen,"The Design and Application of Fluorophore-Gold Nanoparticle Activatable Probes," Physical Chemistry Chemical Physics, Vol. 13, No. 21, 2011, pp. 9929-9941. doi:10.1039/c0cp02967i

[41] Y.-W. Lin, C.-C. Huang and H.-T. Chang, "Gold Nanoparticle Probes for the Detection of Mercury, Lead and Copper Ions," Analyst, Vol. 136, No. 5, 2011, pp. 863871. doi:10.1039/c0an00652a

[42] G. K. Darbha, A. Ray and P. C. Ray, "Gold Nanoparti-
cle-Based Miniaturized Nanomaterial Surface Energy Transfer Probe for Rapid and Ultrasensitive Detection of Mercury in Soil, Water, and Fish," ACS Nano, Vol. 1, No. 3, 2007, pp. 208-214. doi:10.1021/nn7001954

[43] S. Link, Z. L. Wang and M. A. El-Sayed, "Alloy Formation of Gold-Silver Nanoparticles and the Dependence of the Plasmon Absorption on Their Composition," The Journal of Physical Chemistry B, Vol. 103, No. 18, 1999, pp. 3529-3533. doi:10.1021/jp990387w

[44] G. Mie, "Beitrage zur Optik Truber Medien, Spezeill Kolloidaler Metall-sungen,"Annals of Physics, Vol. 25, 1908, pp. 377-455. doi:10.1002/andp.19083300302

[45] D. Ghosh, D. Sarkar and N. Chattopadhyay, "Intramolecular Charge Transfer Promoted Fluorescence Transfer: A Demonstration of Re-Absorption of the Donor Fluorescence by the Acceptor," Journal of Molecular Liquids, Vol. 156, No. 2-3, 2010, pp. 131-136. doi:10.1016/j.molliq.2010.06.006

[46] V. Amendola and M. Meneghetti, "Size Evaluation of Gold Nanoparticles by UV-vis Spectroscopy," The Journal of Physical Chemistry C, Vol. 113, No. 11, 2009, pp. 4277-4285. doi:10.1021/jp8082425

[47] A. L. González, C. Noguez, G. P. Ortiz and G. Rodríguez-Gattorno, "Optical Absorbance of Colloidal Suspensions of Silver Polyhedral Nanoparticles," The Journal of Physical Chemistry B, Vol. 109, No. 37, 2005, pp. 17512-17517. doi:10.1021/jp0533832

[48] A. J. Haes, S. Zou, G. C. Schatz and R. P. Van Duyne, "Nanoscale Optical Biosensor: Short Range Distance Dependence of the Localized Surface Plasmon Resonance of Noble Metal Nanoparticles," The Journal of Physical Chemistry B, Vol. 108, No. 22, 2004, 6961-6968. doi:10.1021/jp036261n

[49] C. A. Mirkin, R. L. Letsinger, R. C. Mucic and J. L. Storhoff, "A DNA-Based Method for Rationally Assembling Nanoparticles into Macroscopic Materials," Nature, Vol. 382, No. 6592, 1996, pp. 607-609. doi: $10.1038 / 382607 \mathrm{a} 0$

[50] G. Jones, W. R. Jackson, C. Y. Choi and W. R. Bergmark, "Solvent Effects on Emission Yield and Lifetime for Coumarin Laser Dyes. Requirements for a Rotatory Decay Mechanism," The Journal of Physical Chemistry, Vol. 89, No. 2, 1985, pp. 294-300. doi:10.1021/j100248a024

[51] P. Sen, D. Roy, S. K. Mondal, K. Sahu, S. Ghosh and K. Bhattacharyya, "Fluorescence Anisotropy Decay and Solvation Dynamics in a Nanocavity: Coumarin 153 in Methyl $\beta$-Cyclodextrins," The Journal of Physical Chemistry A, Vol. 109, No. 43, 2005, pp. 9716-9722. doi:10.1021/jp051607a

[52] D. Seth, D. Chakrabarty, A. Chakrabarty and N. Sarkar, "Study of Energy Transfer from 7-Amino Coumarin Donors to Rhodamine 6G Acceptor in Non-Aqueous Reverse Micelles," Chemical Physics Letters, Vol. 401, No. 4-6, 2005, pp. 546-552. doi:10.1016/j.cplett.2004.11.119

[53] B. N. J. Persson and N. D. Lang, "Electron-Hole-Pair Quenching of Excited States near a Metal," Physical Review B, Vol. 26, No. 10, 1982, pp. 5409-5415. doi:10.1103/PhysRevB.26.5409 